\title{
2015 Wilderness E Environmental Medicine Peer Reviewers
}

\begin{abstract}
The Editors wish to express their sincere appreciation to the following individuals who faithfully and skillfully performed peer reviews for articles submitted to Wilderness \& Environmental Medicine. WEM continues to have a growing impact on the scientific community. We want to acknowledge the importance of our peer reviewers to the process of ensuring the merit and quality of the manuscripts that we publish. Many of these peer reviewers reviewed multiple papers, and some also serve on the Editorial Board and have Section Editor duties as well. Without them we could not produce this high-quality medical journal.
\end{abstract}

John Anderson, USA

Achyut Aryal, New Zealand

E. Wayne Askew, USA

Paul S. Auerbach, USA

Howard Backer, USA*

Jennifer Baine, USA

Anju Bansal, USA

Howard Barkan, USA

Brad L. Bennett, USA*

Jeffrey Bernstein, USA

Aaron R. Billin, USA

Cindy Carol Bitter, USA

Michael Bogdasarian, USA

Lee Bogle, Canada

Adam Booth, UK

Ralph S. Bovard, USA

Steve Boyer, USA

Benjamin Breyer, USA

Aaron Brillhart, USA

Julio Brito, Chile

Naomi Elisabeth Brooks, UK

Hermann Brugger, Italy

Martin Burtscher, Austria

Gabriel Cade, USA

Nigel Callender, UK

Aaron D. Campbell, USA

Michael Cardwell, USA

Eric Carter, Canada

Julie Chase, USA

Anuj Chawla, India

Christopher Cheatham, USA

Kenneth Chiacchia, USA

Kira Christian, USA

Amalia Cochran, USA

Charles Cook, USA

Ed Cornell, Canada

Andrew Creer, USA

Michael Dacre, USA

Eilif Dahl, Norway

Nicholas J. Daniel, USA
Christopher Burney Davis, USA

Kyle P. Davis, USA

Luc de Haro, France

Petar J. DeNoble, USA

Robert W. Derlet, USA

James H. Diaz, USA

Ola Halina Dunin-Bell, Canada

Ron Durheim, USA

Nedim Durmus, Turkey

Bruno Durrer, Switzerland*

Chelsea Dymond, USA

Liz Edelstein, USA

Kurt Power Eifling, USA

Lance S. Ferguson, USA

Scott Forbes, Canada

William Forgey, USA

Joseph Derek Forrester, USA

Tolani Francisco, USA

Flavio G. Gaudio, USA

Lisa-Ann Gershwin, Australia

Torrey Lisa Goodman, USA

Andrea Gravatt, USA

Ian Greatbatch, UK

Mark Greve, USA

Colin K. Grissom, USA*

Abdulkadir Gunduz, Turkey

Susan Haapaniemi, USA

Peter Hackett, USA*

Vidal Haddad, Jr., Brazil

Michael Harrison, USA

Michael Hauty, USA

Tatiana Havryliuk, USA

Seth C. Hawkins, USA

Kristin Jensen Heaton, USA

Carlton Heine, USA

David Hillebrandt, UK

Alastair Hodges, Canada

Tracy Beth Høeg, Denmark

Martin D. Hoffman, USA*

Ulrike Holzgrabe, Germany
David Hostler, USA

Chris Imray, UK

Hillary Irons, USA

Geoffrey K. Isbister, Australia

Kenneth V. Iserson, USA

Michael Jacobs, USA

Dominique Jean, France

Eric Johnson, USA

Barbara Jones, USA

Jeremy Joslin, USA

Nicholas Kanaan, USA

Christopher Kang, USA

Lee Kaplan, USA

Jeffrey Karp, USA

A. Kasturiratne, Sri Lanka

Bengt Kayser, Switzerland

Robert W. Kenefick, USA*

Morteza Khodaee, USA

Kenneth W. Kizer, USA*

Judith Klein, USA*

Pranawa Koirala, Nepal

S.A.M. Kularatne, Sri Lanka

Ricky Langley, USA

Harvey V. Lankford, USA

Stephanie Lareau, USA

Eric John Lavonas, USA

Gemma Lewis, USA

Cynthia R. Lewis-Younger, USA

James Lieberman, USA

Grant S. Lipman, USA*

Felipe Lorenzo, USA

Claude Maillaud, New Caledonia

Sarah Terez Malka, USA

Ryan Mason, USA

Scott Ellis McIntosh, USA

Igor Mekjavic, Slovenia

Debbie Miller, USA

Damian Morgan, Australia

Daniel S. Morris, UK

Michael E. Mullins, USA 
Mária Némethy, USA

Andrew T. Nathanson, USA

Robert Lee Norris, USA

Andrew Nyberg, USA

John Ogle, USA

Kathy Orloski, USA

Edward Joseph Otten, USA*

James A. Padfield, USA

Alberto Enrique Paniz-Mondolfi,

Venezuela

Christopher Pannucci, USA

Yannis Papastamatiou, UK

Avinash Patil, USA

James M. Pattarini, USA

Patrick Peters, Luxembourg

Dan D. Petersen, USA

Alicia Peterson, USA

David Pomeranz, USA

Jessie Poquerusse, USA

Jose Antonio Prieto, Spain

Matiram Pun, Canada*

Robert H. Quinn, USA*

Naeem Quraishi, Pakistan

George W. Rodway, USA*

Ian Rogers, Australia*
Jens Rothenberger, Germany

Edward Ryan, USA

Renee N. Salas, USA

John R. Saucier, USA

Tim Saunders, USA

Kathleen D. Saxon, USA

Volker Schöffl, Germany

Sam Schimelpfenig, USA

Tod Schimelpfenig, USA

Robert Schoene, USA*

Katherine Shea, USA

Suzanne M. Shepherd, USA

David R. Shlim, USA

Arthur Siegel, USA

J. Smith, USA

William R. Smith, USA

Kurt J. Smith, Canada

Susanne J. Spano, USA

John St Cyr, USA

Jennifer Starling, USA

Stasinos Stavrianeas, USA

Christopher R. Stephens, Canada

Matthew J. Stewart, USA

Giacomo Strapazzon, Italy

Kristin Stuempfle, USA
Guenther Sumann, Austria

Douglas Sward, USA

Erik R. Swenson, USA

Karen Van Hoesen, USA

Christopher Van Tilburg, USA

Julian Villar, USA

Scott R. Votey, USA

Dale R. Wagner, USA

Ian Wedmore, USA

Jacob Andreas Wegelin, USA

Lori Weichenthal, USA

Scott Allen Weinstein, Australia*

Robert Weiss, USA

Thomas R. Welch, USA

Albert R. Wheeler, USA

Jenna Marie Bartz White, USA

Matthew Wilson, USA

Edward John Wozniak, USA

Cedric M. Yoshimoto, USA

David Young, USA

Ken Zafren, USA*

*Wilderness \& Environmental Medicine Editorial

Board Member 